\title{
Therapeutic efficacy of AS2077715 against experimental tinea pedis in guinea pigs in comparison with terbinafine
}

\author{
Keisuke Ohsumi, Hidetsugu Murai, Ikko Nakamura, Masato Watanabe and Akihiko Fujie
}

AS2077715 is a novel antifungal metabolite produced by the newly isolated fungal strain Capnodium sp. 339855 . This compound has potent inhibitory activity against Trichophyton mentagrophytes mitochondrial cytochrome $b c_{1}$ complex (complex III) and potent fungicidal activity against $T$. mentagrophytes, as measured in vitro. Here, we compared the effects of AS2077715 and terbinafine in a guinea pig model of tinea pedis. In a treatment regimen started from the day 7 after infection, 10 daily oral doses of 10 and $20 \mathrm{mg} \mathrm{kg}^{-1}$ AS2077715 and $20 \mathrm{mg} \mathrm{kg}^{-1}$ of terbinafine significantly decreased fungal colony-forming units (CFUs) in foot pad skin. In a treatment regimen started from the day 11 after infection, $20 \mathrm{mg} \mathrm{kg}^{-1}$ AS2077715 significantly reduced fungal CFUs in foot pad skin after 7 daily doses in comparison with $20 \mathrm{mg} \mathrm{kg}^{-1}$ terbinafine-treated guinea pigs. Our findings suggest that in vivo potency and efficacy of AS2077715 are equal to or greater than that of terbinafine, positioning AS2077715 as a good candidate for use in treating trichophytosis.

The Journal of Antibiotics (2014) 67, 717-719; doi:10.1038/ja.2014.68; published online 28 May 2014

\section{INTRODUCTION}

Onychomycosis is a common fungal infection of either toenails or fingernails or both and may be due to dermatophytes, yeasts and nondermatophyte molds. ${ }^{1,2}$ In about $90 \%$ of the cases, the cause of onychomycosis is the anthropophilic dermatophytes Trichophyton rubrum or Trichophyton mentagrophytes. ${ }^{3}$ Topical treatment as monotherapy for onychomycosis is not effective, excluding minor cases, due to the poor permeability of the nail plate to topically applied therapeutic agents. ${ }^{4}$ Oral antifungal agents, such as tebinafine and itraconazole, are the most effective agents available to treat onychomycosis. However, these treatments still have high rates of failure (at least 25\%) and relapse incidence $(22.2 \%$ after 3 years $),{ }^{5}$ and potential drug interactions and safety may be an issue. To overcome these problems, a more effective and safer novel antifungal agent is needed. ${ }^{6}$

We previously described a novel anti-Trichophyton antibiotic, AS2077715 (Figure 1), which has potent inhibitory activity against T. mentagrophytes mitochondrial cytochrome $b c_{1}$ complex (complex III) and potent fungicidal activity against T. mentagrophytes measured in vitro. ${ }^{7}$ Here, as the first step in evaluating the potential of AS2077715 as an oral drug for onychomycosis, we examined the anti-Trichophyton activity of orally applied AS2077715 in a guinea pig model of tinea pedis in comparison with terbinafine. In addition, we conducted the preliminary pharmacokinetic study of AS2077715.

\section{MATERIALS AND METHODS}

Planter skin infection models of T. mentagrophytes in guinea pigs Terbinafine was purchased from Tokyo Chemical Industry (Tokyo, Japan). Four-week-old male Hartley guinea pigs were obtained from Japan SLC (Shizuoka, Japan). The plantar portion of the hind foot of the guinea pigs was cleaned using absorbent cotton containing $70 \%$ ethyl alcohol. Clear cotton was then wet with $200 \mu \mathrm{l}$ of T. mentagrophytes FP2103 conidial suspension $\left(1 \times 10^{8}\right.$ spores $\left.\mathrm{ml}^{-1}\right)$, and plaster was used to fix it to the plantar portion of the foot. Test compounds were prepared in $10 \%$ polyoxyethylated hydrogenated castor oil in saline (HCO-60 solution) and orally administered to guinea pigs. Treatment schedules are shown in Figures 2 and 3. Guinea pigs in each group were killed one day after the completion of treatment. The skin of the hind foot was excised and minced with scissors, and each sample was then homogenized in $10 \mathrm{ml}$ sterile saline. The homogenates were diluted and transferred to a 10$\mathrm{cm}$ dish, into which $20 \mathrm{ml}$ Sabouraud dextrose agar medium containing $500 \mu \mathrm{g} \mathrm{ml}^{-1}$ of cycloheximide that had been melted and cooled to $40^{\circ} \mathrm{C}$ was added. The dish was incubated for 3 days at $30^{\circ} \mathrm{C}$, and the colony-forming units (CFUs) per dish were counted. The CFUs per foot were analyzed using Dunnett's multiple comparison test. All animal experimental procedures were approved by the Committee for Animal Experiments of Astellas Pharma.

Pharmacokinetic study in guinea pigs

Test compounds were dissolved in 10\% HCO-60 solution, and were orally administered to guinea pigs ( $n=4$ per test group) at $10 \mathrm{mg} \mathrm{kg}^{-1}$. Blood samples were collected from each guinea pig at $0.5,1,2,4,8$ and $24 \mathrm{~h}$ post administration under anesthetized condition. Liquid chromatography-tandem MS was used to determine plasma concentrations of AS2077715 and terbinafine. The mobile phase consisted of $0.01 \%$ formic acid and acetonitrile 
at ratios of 45:55 (v/v) for AS2077715 and 55:45 (v/v) for terbinafine. The HPLC system and MS/MS system used were an Agilent 1100 (Santa Clara, CA, USA) and a Sciex API-2000 (Framingham, MA, USA), respectively. The analytical column was a WATERS Atlantis column $\left(5 \mu \mathrm{m}, 50 \times 2.0 \mathrm{~mm}^{2}\right.$ inner diameter, Milford, MA, USA) with a flow rate of $0.2 \mathrm{ml} \mathrm{min}^{-1}$. All plasma samples were prepared as follows: a $50 \mu \mathrm{l}$ of plasma was added to a $1.5-\mathrm{ml}$ tube, and then $50 \mu \mathrm{l}$ of $50 \%$ acetonitrile, $10 \mu \mathrm{l}$ of $0.5 \mu \mathrm{g} \mathrm{m}^{-1}$ terbinafine or $10 \mu \mathrm{g} \mathrm{ml}^{-1}$ AS2077715 (as internal standard for each other) and $100 \mu \mathrm{l}$ of acetonitrile were added, and the tube was mixed with a vortex mixer and centrifuged for $5 \mathrm{~min}$ at 14000 r.p.m. The supernatant $(50 \mu \mathrm{l})$ was mixed with $100 \mu \mathrm{l}$ of each mobile phase, and $20 \mu \mathrm{l}$ of this resultant solution was injected into the analytical column. Pharmacokinetic parameters were calcd using a non-compartmental model.

\section{RESULTS}

Antifungal activity of AS2077715 against T. mentagrophytes in a guinea pig model of tinea pedis

Data on T. mentagrophytes CFUs recovered from the foot pad skin of treated guinea pigs are shown in Figure 2. Significantly fewer CFUs<smiles>CCC(C)CC(C)CCC1CCCC(c2c(O)c(C3C(O)C(O)C(O)C3O)cn(C)c2=O)O1</smiles>

Figure 1 Chemical structure of AS2077715.

\section{Treatment schedule}

Days after infection

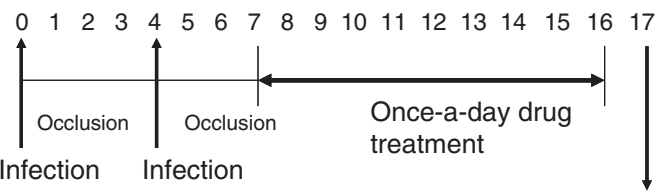

Culture study

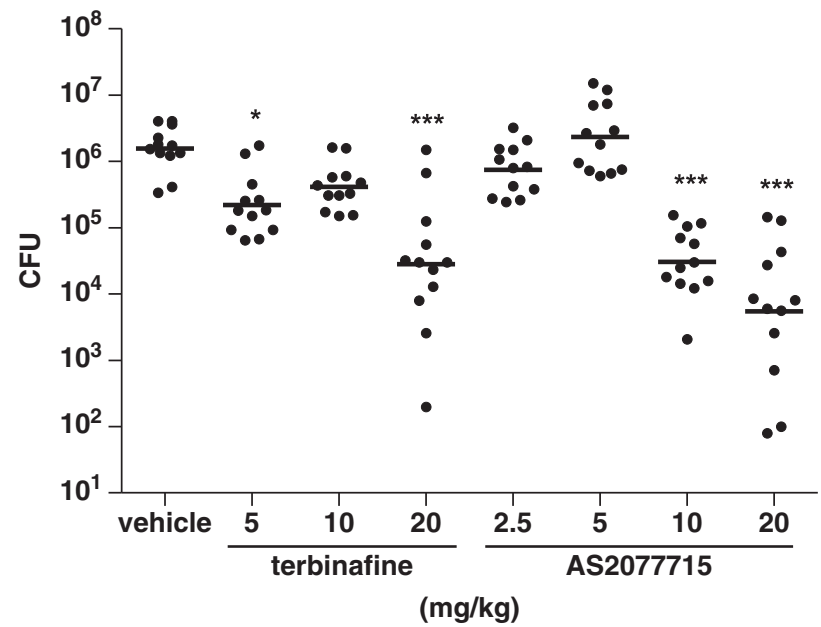

Figure 2 Scattergrams of the number of colony-forming unit (CFU) of T. mentagrophytes recovered from the foot pad skins of guinea pigs treated with terbinafine and AS2077715 in a tinea pedis model. ${ }^{* * *} P<0.001$ versus the vehicle-treated group; ${ }^{*} P<0.05$ versus the vehicle-treated group. were observed in the foot pads of guinea pigs treated with $20 \mathrm{mg} \mathrm{kg}^{-1}$ terbinafine $(P<0.001)$ than in vehicle-treated animals, whereas no significant differences were noted in CFUs observed in the foot pads of guinea pigs receiving a dose of $10 \mathrm{mg} \mathrm{kg}^{-1}$ terbinafine versus vehicle-treated animals. Treatment with AS2077715 significantly reduced CFUs at 10 and $20 \mathrm{mg} \mathrm{kg}^{-1}(P<0.001)$ compared with vehicle-treated animals, and the degree of reduction was equivalent to the reduction seen in animals receiving $20 \mathrm{mg} \mathrm{kg}^{-1}$ terbinafine.

To compare the fungal clearance efficacy between AS2077715 and terbinafine in this model, treatments were started from the day when pathology on the skin was present (the 11th day post infection). Further, to investigate the onset of effect, colony-forming assays were performed at several time points. Treatment with $20 \mathrm{mg} \mathrm{kg}^{-1}$ AS2077715 significantly reduced the CFUs in the skin of treated animals after seven daily doses compared with vehicle-treated guinea pigs $(P<0.001)$ and after seven daily doses of AS2077715 (at day 18), the degree of reduction in CFUs was superior to terbinafine $(P<0.001)$ (Figure 3). However, after 14 daily doses of AS2077715, the degree of reduction in CFUs was equal to terbinafine. AS2077715 was therefore deemed equipotent to terbinafine but with earlier demonstration of therapeutic efficacy.

\section{Pharmacokinetics in guinea pigs}

The plasma concentrations of terbinafine and AS2077715 in guinea pigs after oral administration of a single $10 \mathrm{mg} \mathrm{kg}^{-1}$ dose are shown in Figure 4, and pharmacokinetic and pharmacodynamic parameters are summarized in Table 1. The time above MIC (T $>$ MIC) and the area under the curve (AUC)/MIC value of AS2077715 were lower than that of terbinafine.

Treatment schedule
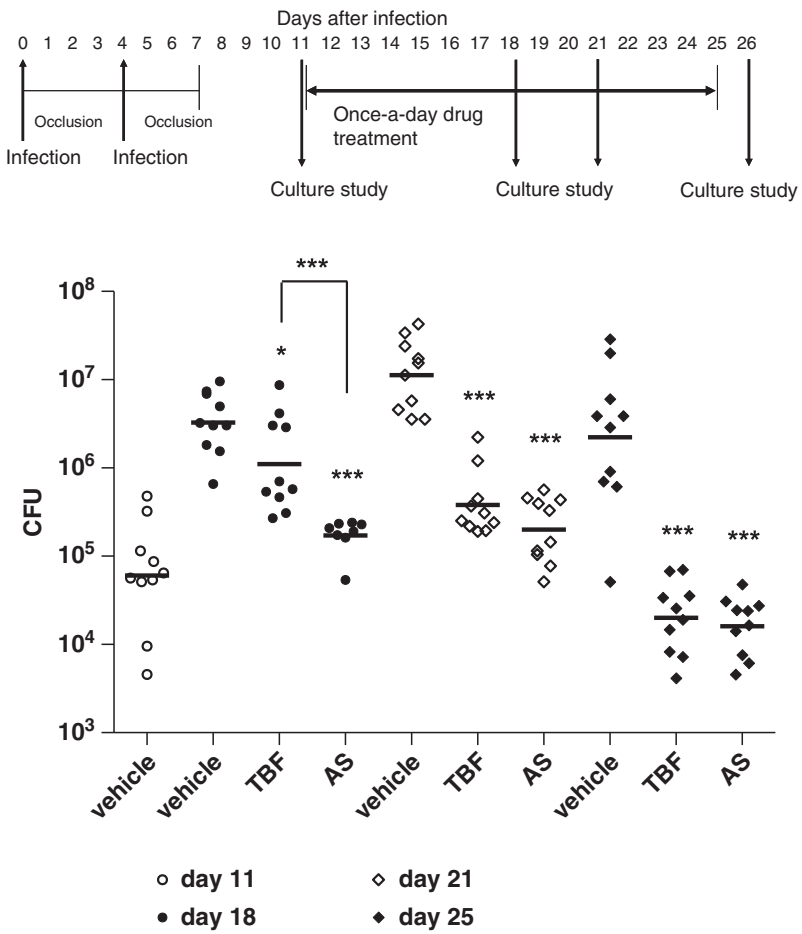

Figure 3 Scattergrams of the number of colony-forming unit (CFU) of T. mentagrophytes recovered from the foot pad skins of guinea pigs treated with terbinafine and AS2077715 at a dose of $20 \mathrm{mg} \mathrm{kg}^{-1}$ in a tinea pedis model. ${ }^{* * *} P<0.001$ versus the vehicle-treated group; ${ }^{*} P<0.05$ versus the vehicle-treated group. 


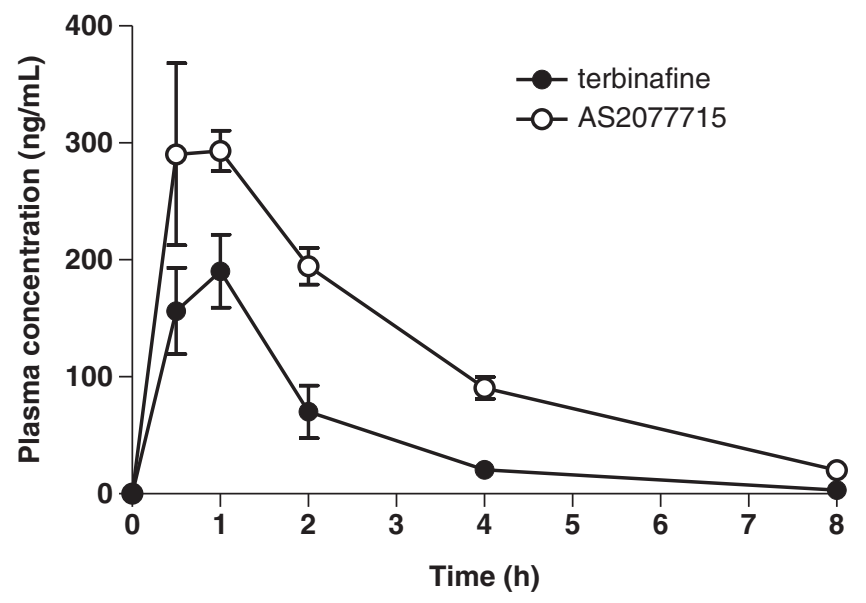

Figure 4 Mean plasma concentrations of AS2077715 and terbinafine in guinea pigs after oral administration of a single dose $\left(10 \mathrm{mg} \mathrm{kg}^{-1}\right)$; error bars depict s.e.m.

Table 1 Pharmacokinetic and pharmacodynamic parameters of AS2077715 and terbinafine in guinea pigs after oral administration of a single $10 \mathrm{mg} \mathrm{kg}^{-1}$ dose (mean \pm s.d.)

\begin{tabular}{lcccccc}
\hline $\begin{array}{l}\text { Test } \\
\text { substrates }\end{array}$ & $\begin{array}{c}\mathrm{C}_{\max } \\
\left(n g m l^{-1}\right)\end{array}$ & $\mathrm{T}_{\max }(h)$ & $\begin{array}{c}\text { AUC } \mathrm{C}_{0-24 h} \\
\left(n g h \mathrm{Il}^{-1}\right)\end{array}$ & $\begin{array}{c}\text { MIC } \\
\left(n g m l^{-1}\right)\end{array}$ & $\begin{array}{c}\text { T>MIC } \\
(h)\end{array}$ & $\begin{array}{c}\text { AUC/MIC } \\
\left(n g h m l^{-1}\right)\end{array}$ \\
\hline AS2077715 & $338 \pm 95$ & $0.8 \pm 0.3$ & $1141 \pm 32$ & 80 & around 4 & 14 \\
Terbinafine & $194 \pm 53$ & $0.8 \pm 0.3$ & $423 \pm 13$ & 10 & around 6 & 42 \\
\hline
\end{tabular}

Abbreviations: $\mathrm{C}_{\max }$, maximum concentration; $\mathrm{T}_{\max }$, time to $\mathrm{C}_{\max }$; $\mathrm{AUC}$, area under the concentration-time curve from zero up to $24 \mathrm{~h}$; MIC, MIC for T. mentagrophytes FP2103, T>MIC, time above MIC.

\section{DISCUSSION}

In a previous report, we demonstrated that AS2077715 had potent in vitro antifungal activity against T. mentagrophytes FP2103. ${ }^{8}$
Here, we examined the anti-Trichophyton activity of orally administered AS2077715 in a guinea pig model of tinea pedis in comparison with terbinafine and found that AS2077715, at doses of 10 and $20 \mathrm{mg} \mathrm{kg}^{-1}$, was effective at reducing fungal burden in plantar skin of guinea pigs, while terbinafine was effective at $20 \mathrm{mg} \mathrm{kg}^{-1}$ but not at $10 \mathrm{mg} \mathrm{kg}^{-1}$ (Figure 2). In addition, the treatment period of AS2077715 necessary to show therapeutic effect was shorter than that of terbinafine (Figure 3). In contrast, MIC of AS2077715 against T. mentagrophytes was eight times higher than terbinafine; ${ }^{8}$ therefore, pharmacodynamic parameters ( $\mathrm{T}>\mathrm{MIC}$ and AUC/MIC) of AS2077715 in guinea pigs were lower than those of terbinafine (Table 1). AS2077715 was superior to terbinafine in terms of its early killing effect against T. mentagrophytes. ${ }^{7}$ Fungicidal activity seems to be more important than MIC for the reduction of fungal burden in planter skin infected with T. mentagrophytes.

In conclusion, AS2077715 exhibits a therapeutic effect equal to or better than terbinafine in guinea pigs, making it a promising candidate for the treatment of trichophytosis. Assessment of the potential of this compound for use against onychomycosis will require evaluation of the effect in a nail infection model.

1 Ramesh, V., Reddy, B. S. \& Singh, R. Onychomycosis. Int. J. Dermatol. 22, 148-152 (1983).

2 Zaias, N. Onychomycosis. Arch. Dermatol. 105, 263-274 (1972).

3 Elewski, B. E. Onychomycosis: pathogenesis, diagnosis, and management. Clin. Microbiol. Rev. 11, 415-429 (1998).

4 Kaur, I. P. \& Kakkar, S. Topical delivery of antifungal agents. Expert. Opin. Drug. Deliv. 7, 1303-1327 (2010).

5 Hay, R. J. The future of onychomycosis therapy may involve a combination of approaches. Br. J. Dermatol. 145(Suppl 60), 3-8 (2001).

6 Arrese, J. E. \& Pierard, G. E. Treatment failures and relapses in onychomycosis: a stubborn clinical problem. Dermatology 207, 255-260 (2003).

7 Ohsumi, K., Watanabe, M. \& Fujie, A. AS2077715 is a selective inhibitor of fungal mitochondrial cytochrome $b c_{1}$ complex. J. Antibiot. 67, 713-716 (2014).

8 Ohsumi, K., Masaki, T., Takase, S., Watanabe, M. \& Fujie, A. AS2077715: a novel antifungal antibiotic produced by Capnodium sp. 339855. J. Antibiot. 67, 707-711 (2014). 